\title{
Using Fuzzy Logic to Construction of Expert Computer Model for Forecast of Compressive Strength of the Portland Cement
}

\section{Basil Y. Al-Khayyat \\ Sufyan S. Al-Dabbagh}

College of Computer Science and Mathematics

University of Mosul, Iraq

\section{Received on: 02/09/2004}

Accepted on: 05/04/2005

\section{ABSTRACT}

The present study aims at construction of expert computer model for forecast of compressive strength of the Portland cement. This model is achieved by chemical data input through interface designed by matlab program. This is followed by calculation of the content of phases; namely, $\mathrm{C}_{3} \mathrm{~S}, \mathrm{C}_{2} \mathrm{~S}, \mathrm{C}_{3} \mathrm{~A}$ and $\mathrm{C}_{4} \mathrm{AF}$. Later on , connection with fuzzy logic interface were made. The formentioned phases represent the input to the fuzzy logic interface through the condition rules IF-THEN. After defuzzification, the values of cement compressive strength appeared on the interface. The evaluations were made through calculation of square difference of the results of fuzzy logic ( present study) and statistical methods.

Keywords: fuzzy logic, expert computer model.

$$
\begin{aligned}
& \text { استخدام المنطق المضبب في تصميم نظام حاسوبي خبير للتنبؤ } \\
& \text { بقوة مقاومة انضغاط الأسمنت البورتلندي } \\
& \text { سفيان سالم الاباغ } \\
& \text { باسل يونس ذنون الخياط } \\
& \text { كلية علوم الحاسبات والرياضيات، جامعة الموصل } \\
& \text { تاريخ قبول البحث: 2005/04/05 } \\
& \text { تاريخ استلام البحث: 2004/09/02 } \\
& \text { الملخص } \\
& \text { تهـدف الدراسـة الحاليـة إلى تصـيم نظـام حاسوبي خبيـر للتبؤ بقوة مقاومـة انضـغاط }
\end{aligned}
$$

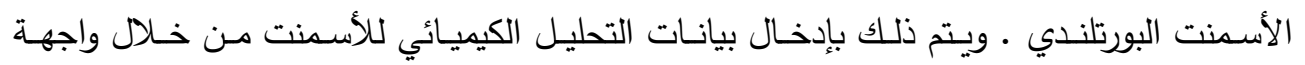

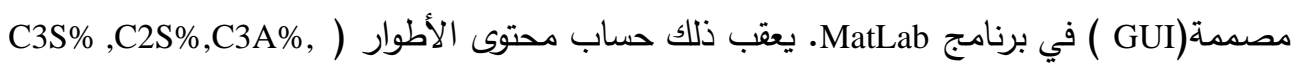

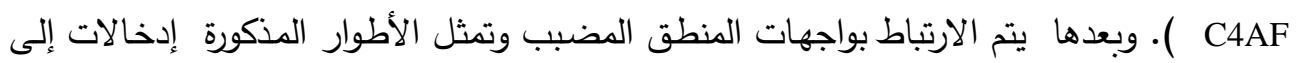

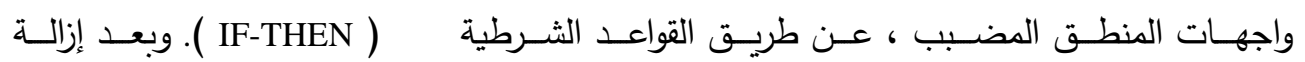

$$
\begin{aligned}
& \text { الضبابية (Defuzzification) تظهر على الواجهة قيمة قوة مقاومة الانضغاط للأسمنت ـ ويتم تقييم } \\
& \text { نتائج المنطق المضبب بمقارنتها مع نتائج الطرائق الإحصائية ومن خلال حساب معدل مربع الفرو } \\
& \text { قات لنتائج كلتا الطريقتين. }
\end{aligned}
$$

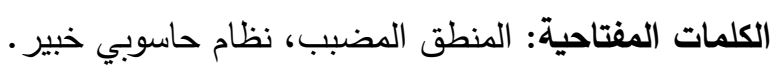




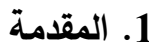

مهما بلغ التطور الكبير في معدات الإنتاج ووحدات التحكم الإكترونية و أعمال السيطرة

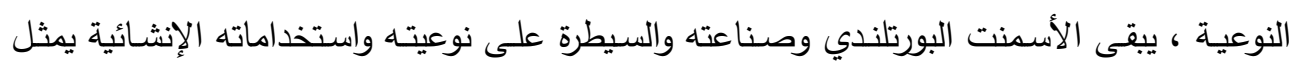

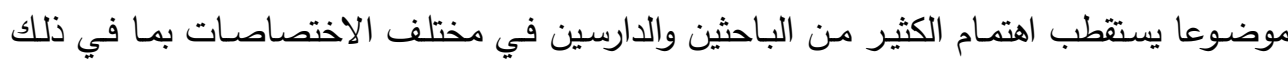

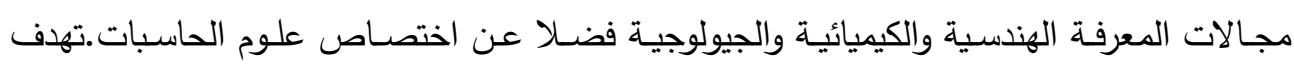

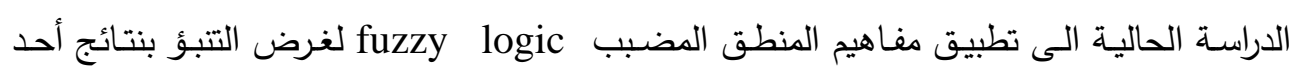

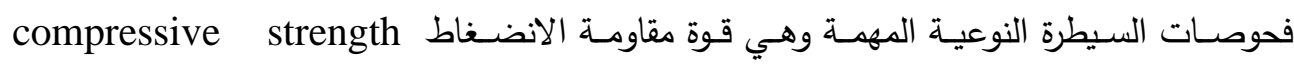

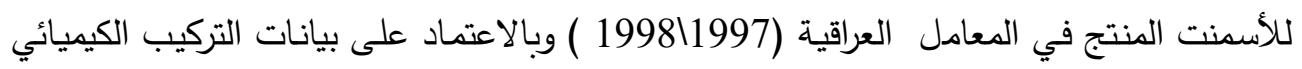

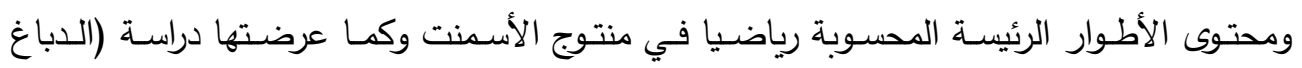
والمعاضيدي ،2004) و ( المعاضيدي والدباغ، 2001 ) . تعتمد صناعة الأسمنت البورتلندي على مواد أولية تتمثل بالأساس من مزيج من نولاع، موعين

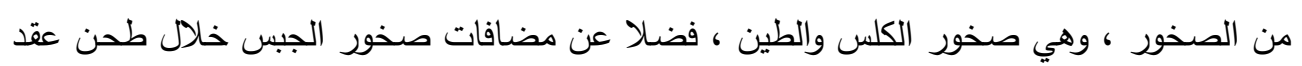

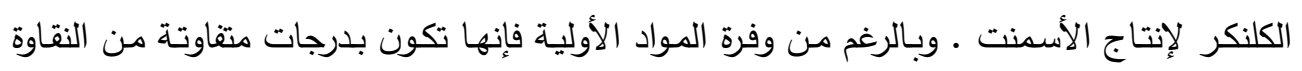

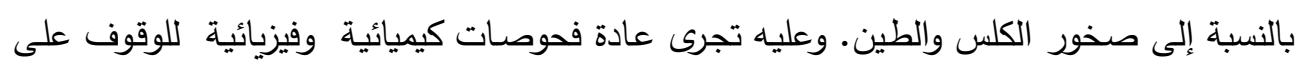

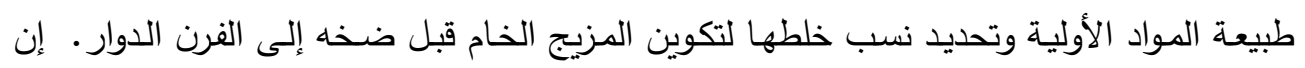

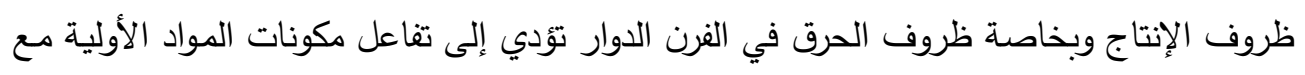
بعضها البعض وتكوين أطوار جديدة تكون مسؤولة عن أداء الأسمنت البورتلندي كمادة إنشائية ،

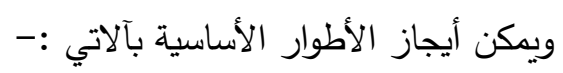
أ-الايت :- وتطلق هذه التسمية على الطور غير النقي من سيلكات الكالسيوم الثلاثي أما الطور النقي فيرمز له(c3s) • ولهذا الطور ستة أشكال ومنها ثلاثة بنظام بلوري ثلاثي الميل واثنان أحاديا

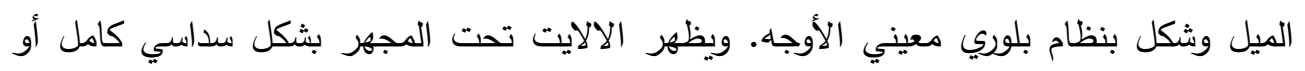

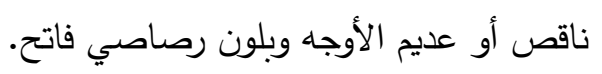

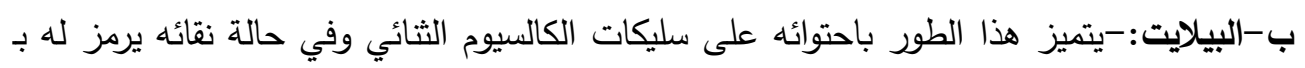

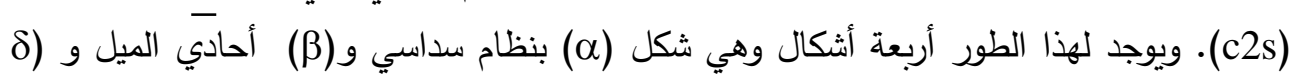

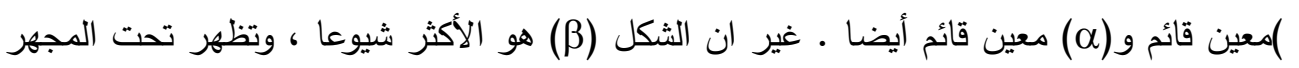

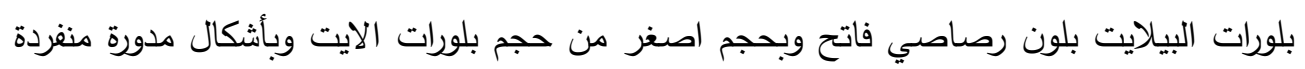
أو بشكل حشود مفككة أو بشكل تجمع عنقودي. 
ج.--الامومينيت :- يحتوي هذا الطور في الأساس على الومينات الكالسيوم الثلاثي ويرمز للحالة النقية منه بالرمز (C3A) • ويشارك الالومينيت في أطوار مادة الحشو التي توجد بأحجام ناعمة

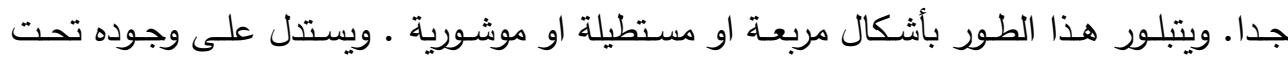
المجهر من خلال قابليته العالية على التحفر عند معالجته بالماء.

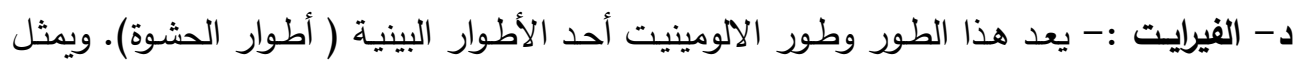
الفيرايت الطور الرئيس الحامل للحديد ويتكون من الومينات حديد الكالسيوم الرباعي ويرمز للحالة

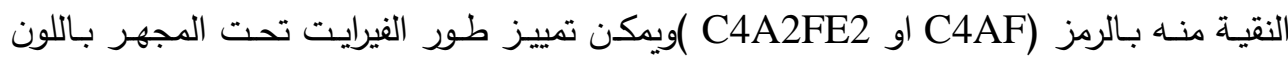
الرصاصي الفاتح - الأبيض أو بانعكاسية عالية للضوه. هـ - الأطوار الأخرى :- تشير ملاحظات الفحص المجهري إلى وجود أطوار أخرى بكميات قليلة

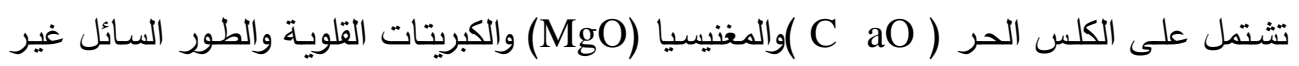
المجزء ( طور الزجاج ) فضلا عن الفراغات الناتجة عن هروب الغازات خلال الحرق.

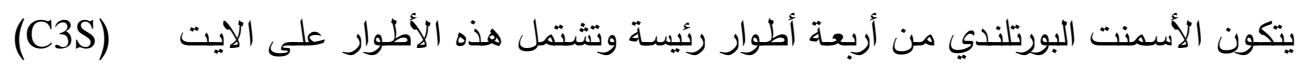

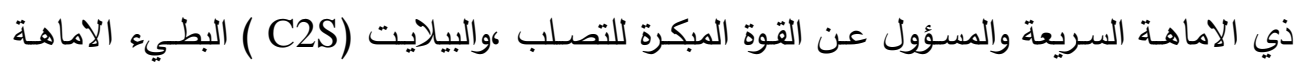
والمسؤول عن القوة السريعة جدا، وطور الالومينيت (C3A) ذي الاماهـة السريعة جدا وطور الفيرايت (C4AF) ذي الاماهـة البطيئة جدا. والطوران الأخيران غير مسؤولين عن قوة تصلب الأبئ

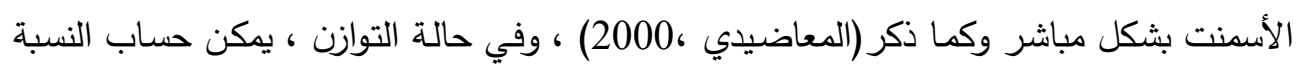

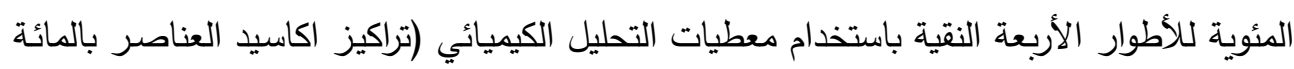
وزنا :- , SiO2 , Al2O3 , Fe2O3 , CaO , SO3 فضـا عن طور سليكات الكالسيوم

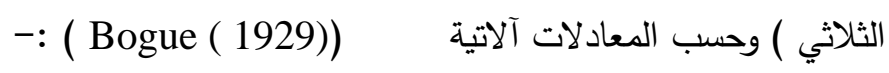

$\mathrm{C}_{3} \mathrm{~S}=4.071(\mathrm{CaO}-$ free lime $)-7.6 \mathrm{SiO}_{2}-6.71 \mathrm{Al}_{2} \mathrm{O}_{3}-1.43 \mathrm{Fe}_{2} \mathrm{O}_{3}-2.85 \mathrm{So}_{3}$.

$\mathrm{C}_{2} \mathrm{~S}=2.867 \mathrm{SiO}_{2}-0.754 \mathrm{C}_{3} \mathrm{~S}$.

$\mathrm{C}_{3} \mathrm{~A}=2.650 \mathrm{Al}_{2} \mathrm{O}_{3}-1.692 \mathrm{Fe}_{2} \mathrm{O}_{3}$.

$\mathrm{C}_{4} \mathrm{AF}=3.04 \mathrm{Fe}_{2} \mathrm{O}_{3}$.

يخضع وبشكل روتيني الأسمنت البورتلندي المنتج في جميع المعامل لفحوصات السيطرة

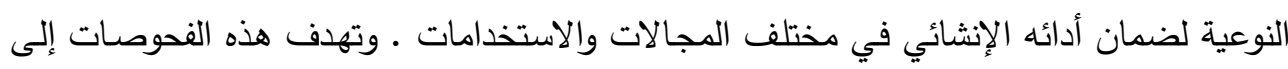

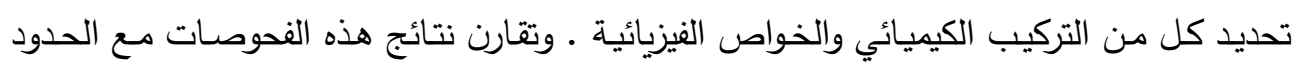

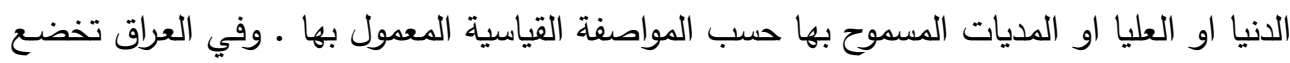


فحوصات السيطرة النوعية للمواصفة القياسية رقم (5) لسنة 1984. وتثمل هذه المواصفة التحاليل الكيميائية وحساب النسبة المئوية للأطوار الرئيسة وبعض المعاملات الدالة (ذنون ، 1979).

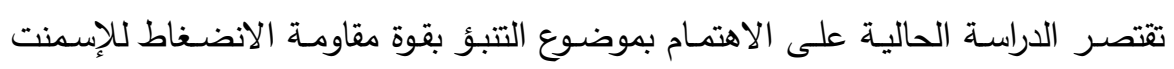
البورتلندي سـواء أَكان ذلك باستخدام الطرق الإحصـائية ( الرياضـية) أم بالاعتمـاد على مفـاهيم

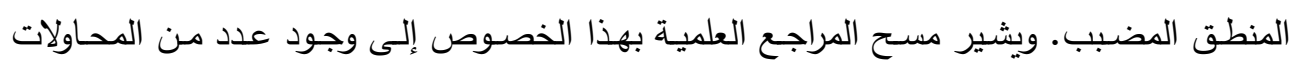

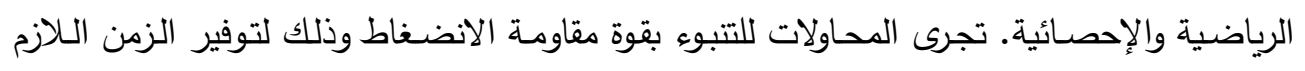

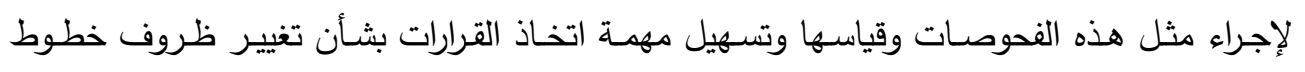

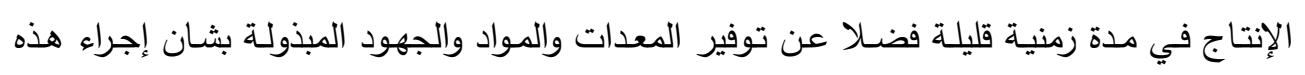
الفحوصات.

لقد حاول (Kohlhaas , 1983 ) إيجاد العلاقة الرياضية بين قوة مقاومة الانضغاط

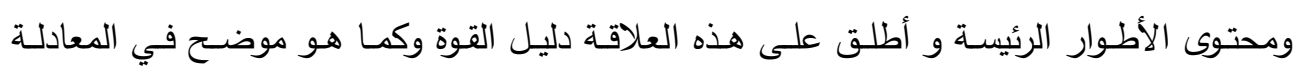

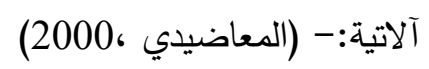

$\mathrm{F} 28=(3$ alite $)+(2$ belite $)+($ aluminate $)$-ferrite

ويجدر التتويـه هنـا بإن تطبيق هذه المعادلة على بيانات الدراسة الحالية يؤدي الى قيم مشوهة من قوة مقاومة الانضغاط للإسمنت البورتلندي العراقي.

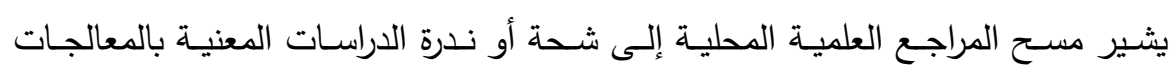

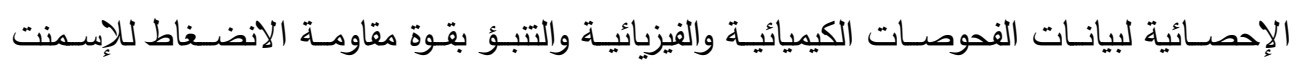
البورتلندي العراقي (المعاضيدي ،2000). وربما يعود السبب في ذلك إلى محدودية تداول بيانات

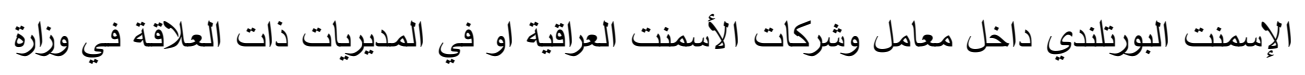
الصناعة والمعادن.

تعـد دراسـة (ذنـون ،1979) مـن أوائـل الدراسـات عـن المعالجـات الإحصـائية لبيانـات

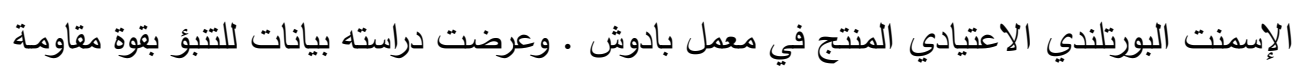

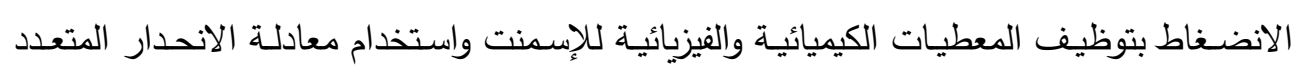

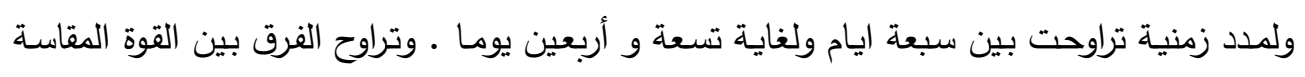

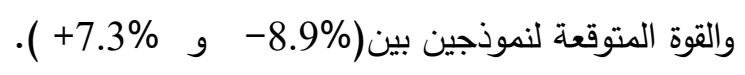

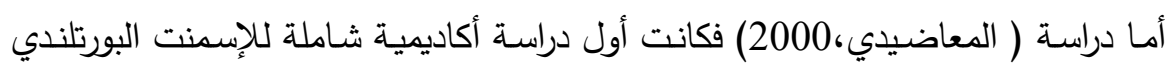

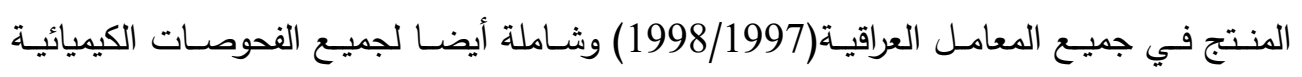


والفيزيائية بما في ذلك الفحوصات المجهرية والمعدنية فضلا عن المعالجات الإحصائية لمعطيات الدراسة ـ وبسبب هذه الميزات اعتمدت الدراسة الحالية على بيانات ومعطيات دراسة (المعاضيدي

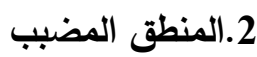

إن المنطق المضبب هو تقنية تتمتع بقدرة عالية على إيجاد الحلول للمشاكل المختلفة بما

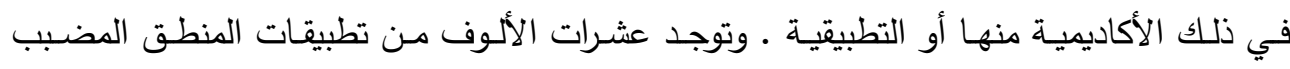
الداخلة ضمن عمليات السيطرة والمعلوماتية ـ ويوفر المنطق المضبب طريقة بسيطة جدا للحصول

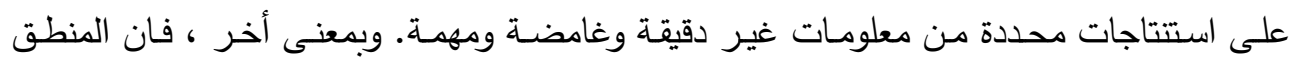

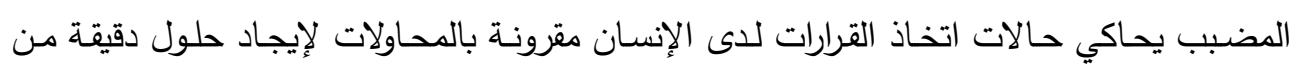

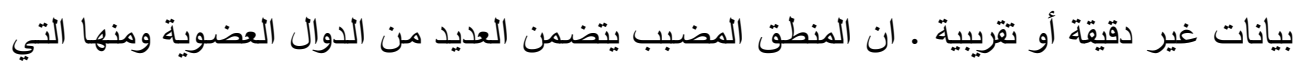

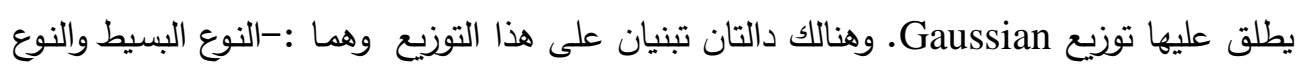

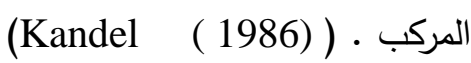

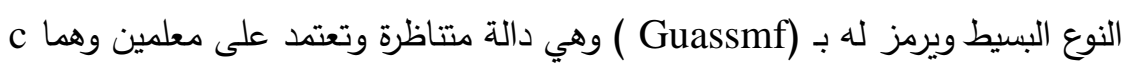
ورة وكما في المعادلة الآتية :-

$$
\mu(x)=e^{\frac{-(x-c)^{2}}{2 * \partial^{2}}}
$$

أمـا النوع المركب ويرمز لـه بـ (Guass2mf ) وهذه الدالة هي دمـج لدالتين من نوع

$\cdot($ Guassmf)

إن المنطق المضبب يعتمد أساسـا على مجموعة من قواعد الأقسام الثرطية بشكل إذا-

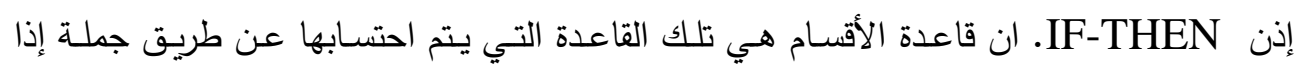

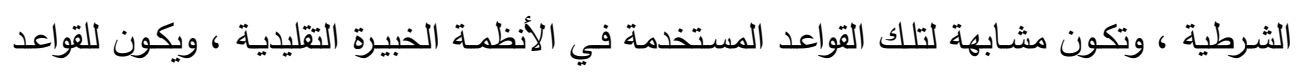

if $w$ is $Z$ then $x$ is $Y$

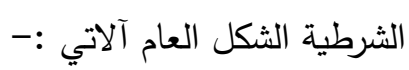

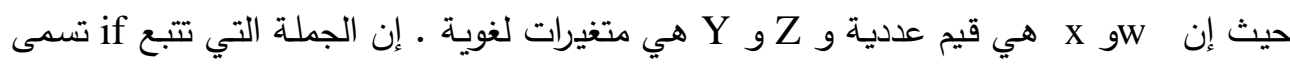

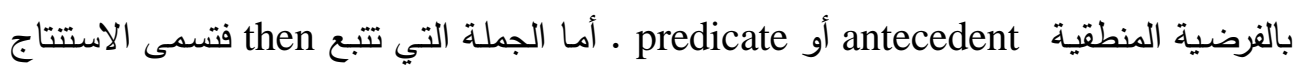
consequent تتنمي X إلى Y بنفس درجة عضوية W إلى Z . . (حمودات (2002) ) 


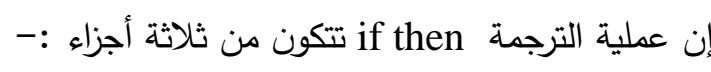

أ.الإدخال المضبب ( fuzzy input ).

ب.تطبيق عملية مضبية على الأجزاء المتعددة (apply fuzzy operator to multiple parts).

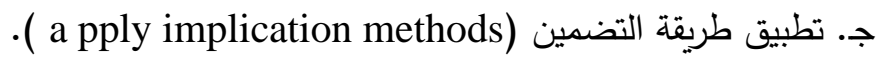

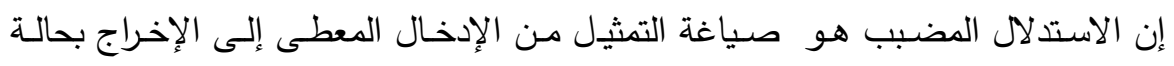

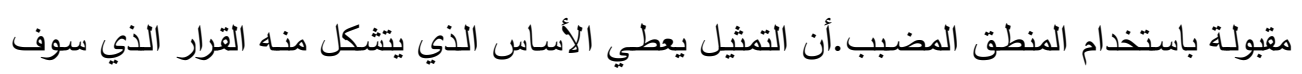

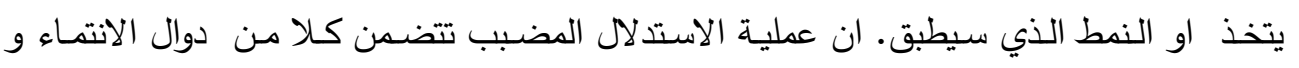

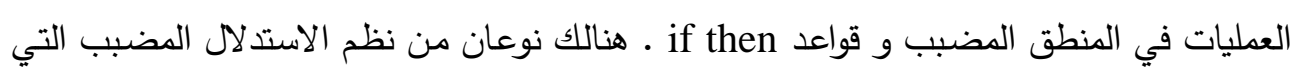

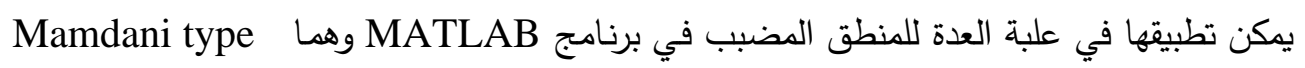

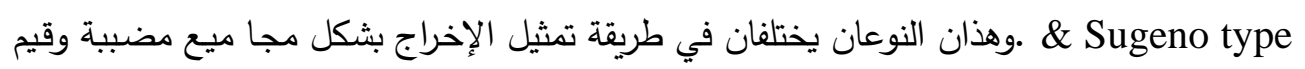

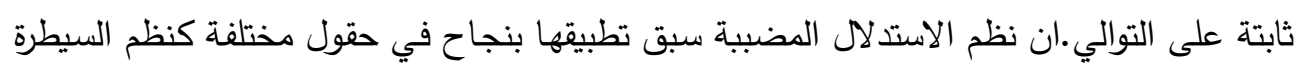

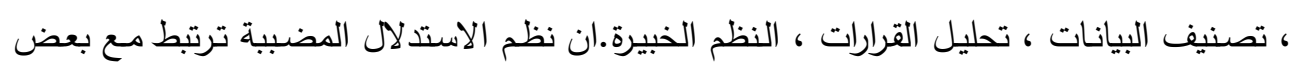

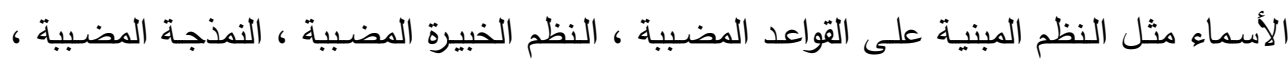

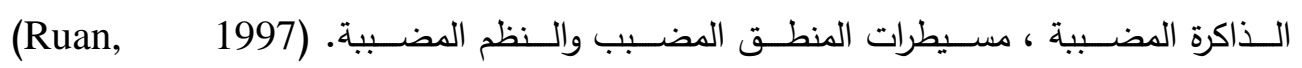
و (Zadeh,1995) إن مميزات نظم الاستدلال من نوع مامداني تكون مقبولة بشكل واسع وملائمة للإدخالات

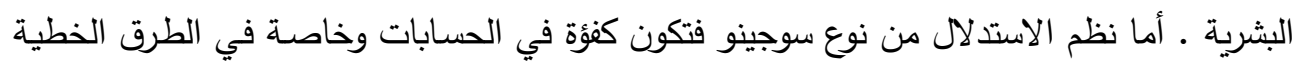

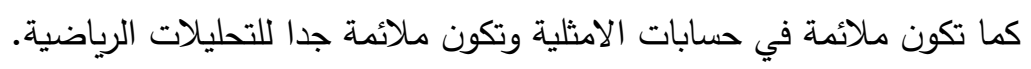
3. بيانات التحليل الكيميائي و الفحوصات الفيزيائية

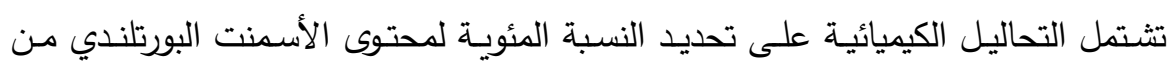

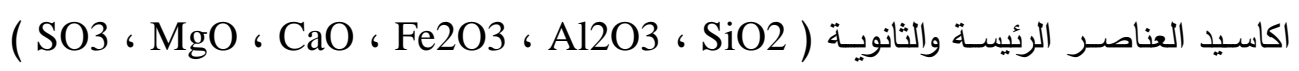

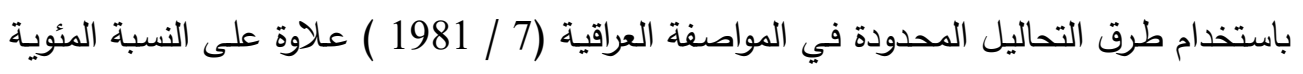

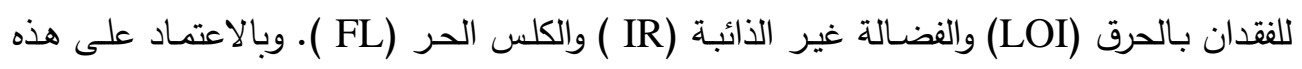

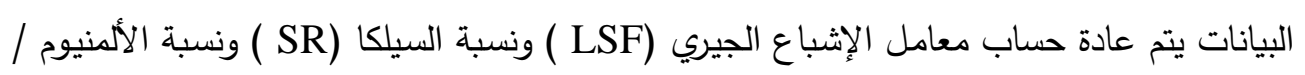

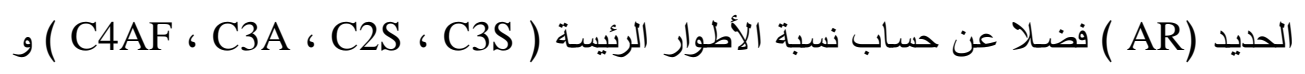

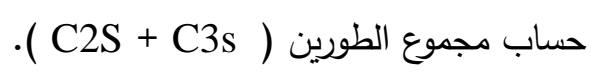


تجرى عـادة الفحوصـات الفيزيائيسة بموجب المواصفة العراقيـة (1968/8) وتشـمل على

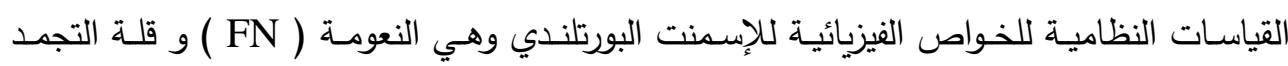
الابتدائي والنهائي ( I.ST و F.ST ) وقوة مقاومسة الانضـغاط لثلاثتة ايـام ( 3D ) وسـبعة أيسام

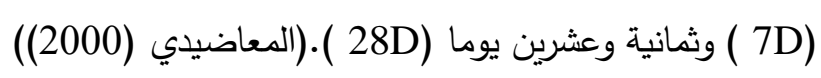

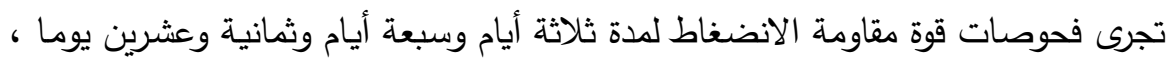

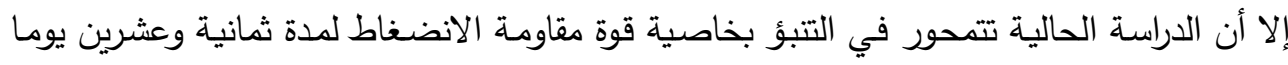

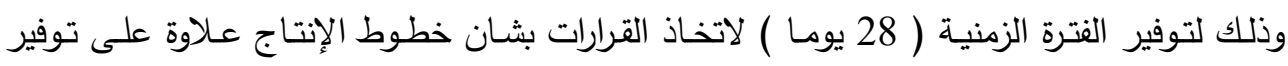

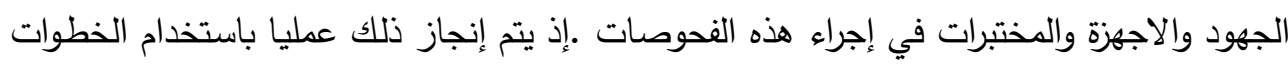
المؤشرة في المواصفة القياسية العراقية رقم (5) لسنة 1984. 4. استخدام المنطق المضبب لتقدير قوة مقاومة انضغاط الأسمنت تم الاعتماد على بيانات التحليل الكيميائي و الفحوصات الفيزيائية باستخدام الجداول آلاتية: الجدول (1) مديات تراكيز اكاسيد العناصر

\begin{tabular}{|c|c|}
\hline اساسيد العناصر & $\mathrm{SiO} 2$ \\
\hline $23.72-21.21$ & $\mathrm{Al2O} 3$ \\
\hline $5.88-5.16$ & $\mathrm{Fe} 2 \mathrm{O} 3$ \\
\hline $4.01-1.75$ & $\mathrm{CaO}$ \\
\hline $63.74-58.33$ & $\mathrm{SO} 3$ \\
\hline $4.18-1.76$ & Free lime \\
\hline $2.91-0.56$ & \\
\hline
\end{tabular}

جدول (2) مديات محتوى الأطوار الرئيسة

\begin{tabular}{|c|c|}
\hline الأطوار الرئيسة \\
\hline $39.3-10.6$ & C3S \\
\hline $58.3-34.9$ & C2S \\
\hline $12.4-6.9$ & C3A \\
\hline $12.2-5.3$ & C4AF \\
\hline
\end{tabular}




\begin{tabular}{|c|c|}
\hline \multicolumn{2}{|c|}{ الجدول (3) مديات نتائج الفحوصات الفيزيائية } \\
\hline 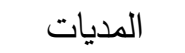 & الفحوص الفيزيائية \\
\hline $29.0-14.5$ & قوة مقاومة الأسمنت (3 أيام) \\
\hline $37.3-23.0$ & قوة مقاومة الأسمنت (7 أيام) \\
\hline $45.5-25$ & قوة مقاومة الأسمنت (28 يوماً) \\
\hline
\end{tabular}

يتضمن هذا البحث دراسة العلاقة بين كل من الأطوار الأبعة الرئيسة ( C3S و C2S

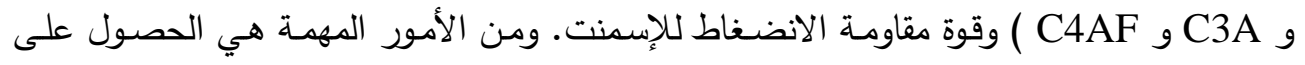
منحنيات ممهدة smooth curves توضح العلاقة بين كل من الأطوار الرئيسة الأربعة وقوة مقاومة الأدورة

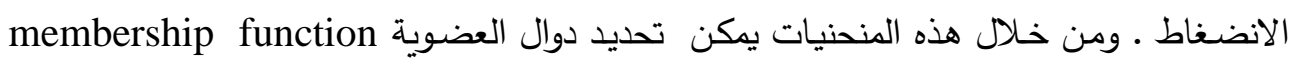
واللازمة لبناء النظام الحاسوبي المضبب. ومن الامور المهمة التي تمكننا من بناء نظام حاسوبي

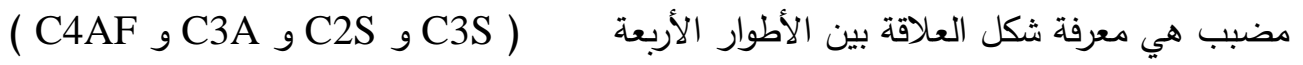
وقوة مقاومة الانضغاط للإسمنت ، إذ انه من شكل العلاقة يمكن تحديد دالة العضوية المناسبة في كل حالة .

Scatter Diagram في البداية تم استخدام الأسلوب الكلاسيكي المعروف بشكل الانتشار

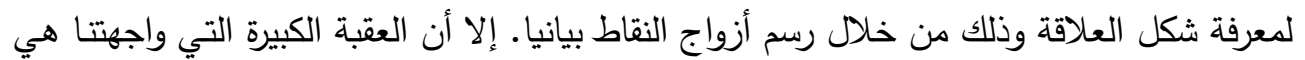

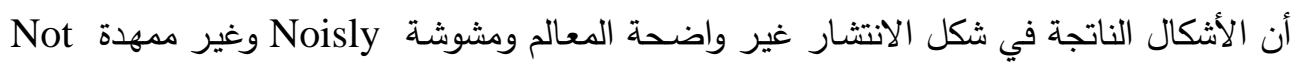

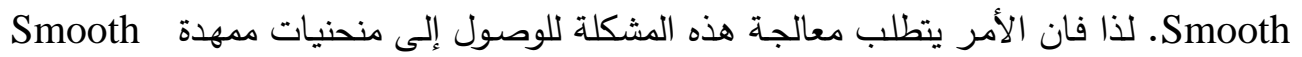
Curves

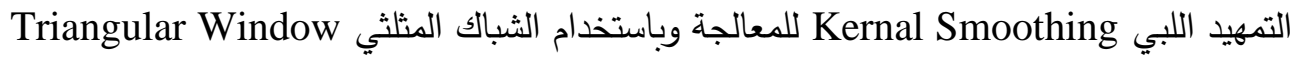
(ذنون ،1998) .وتم إعداد البرامج اللازمة لهذه الطريقة باستخدام تطبيق MatLab كي يتم ربطها

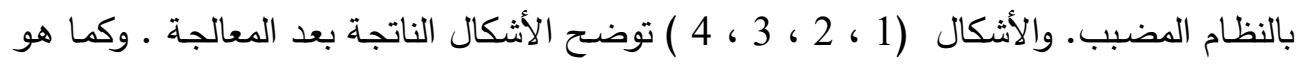

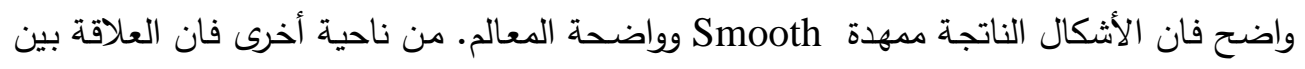
قوة انضغاط الأسمنت وكل طور من الأطوار الأربعة هي علاقة غير خطية لها شكل خاص بها فها ،

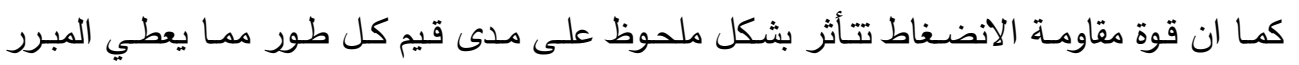

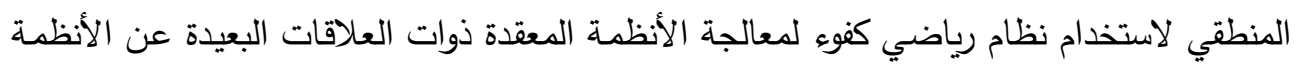

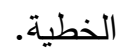




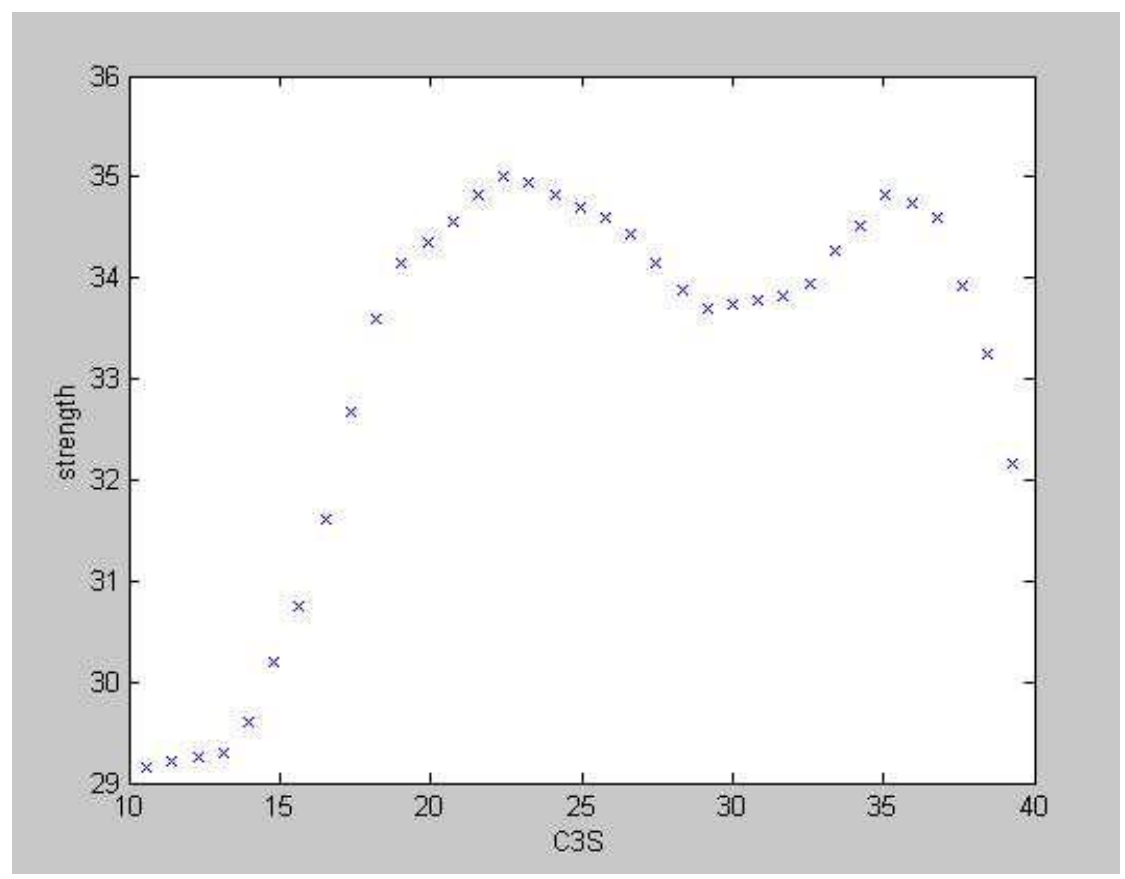

الشكل (1) العلاقة بين C3S وقوة الانضغاط بعد التمهيد

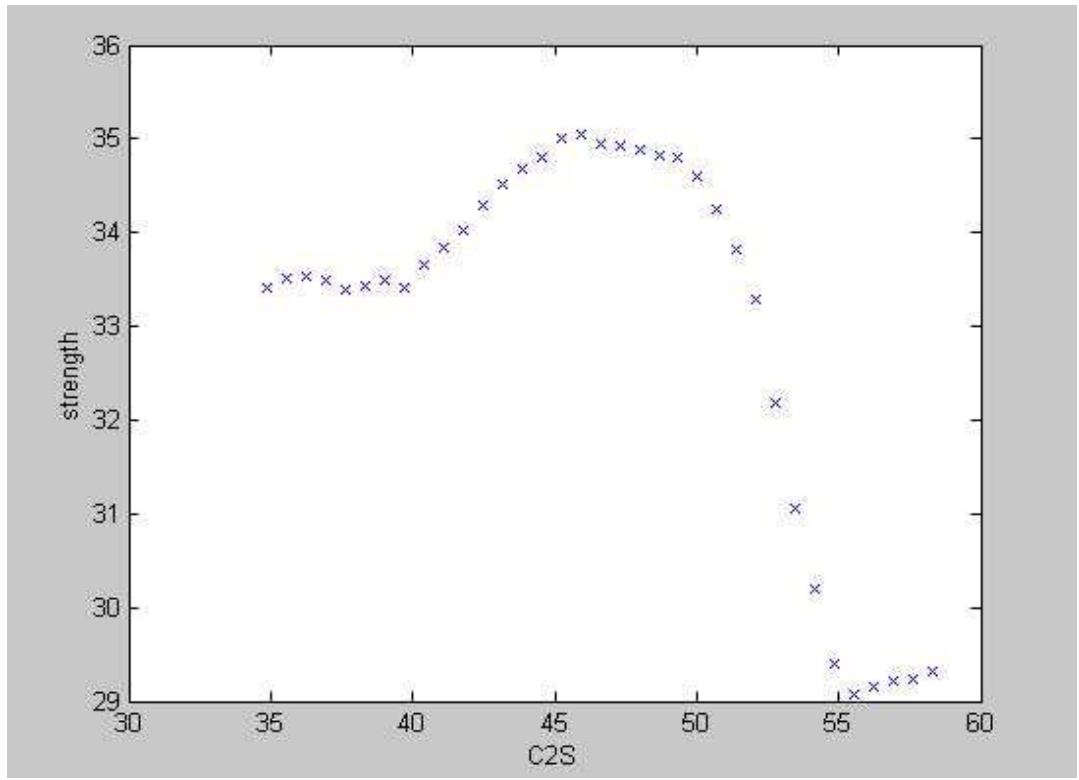

الشكل (2) العلاقة بين C2S وقوة الانضغاط بعد التمهيد 


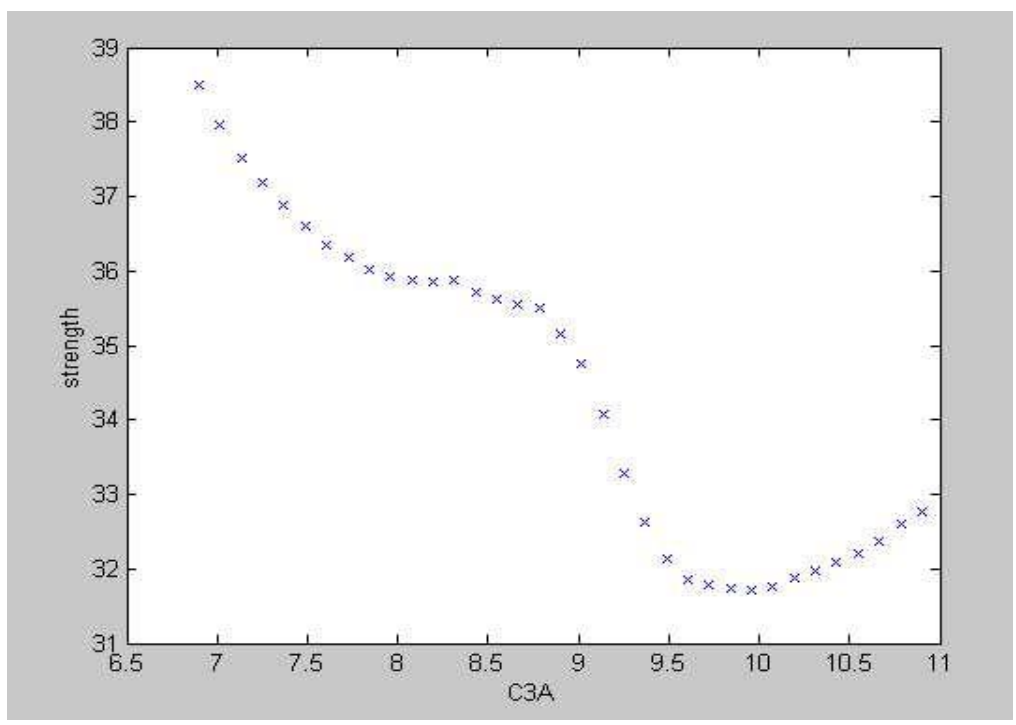

الثكل (3) العلاقة بين C3A وقوة الانضغاط بعد التمهيد

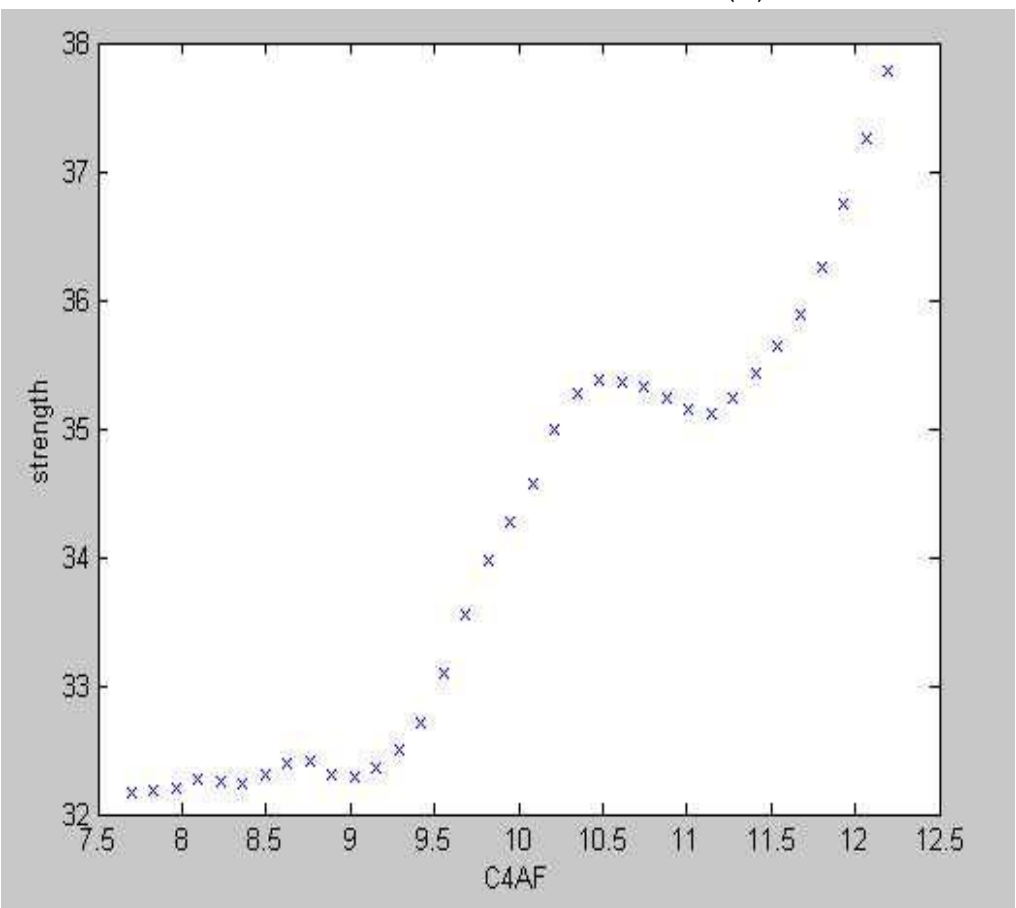

الثكل (4) العلاقة بين C4AF وقوة الانضغاط بعد التمهيد 
لقد تم تصميم نظام حاسوبي مضبب يقوم بفحص الخواص الفيزيائية والكيميائية للإسمنت ومطابقة هذه الخواص للمواصفة العالمية ومن ثم تقدير قوة مقاومة انضغاط الأسمنت. وتم الاعتماد

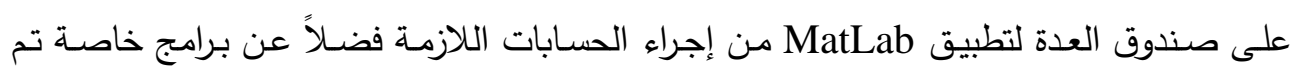
ربطها بالنظام لغرض إكمال عمليات التمهيد اللازمة.

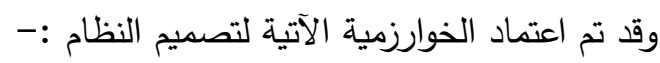

الخطوة الأولى :- ندخل البيانات الخام على واجهات مصممة وهذه البيانات تمثل التحاليل الفيزيائية والكيميائية للإسمنت.

الخطوة الثانية :- يتم حساب قيم الأطوار الأربعة باستخدام معادلات تسمى معادلات بوك .

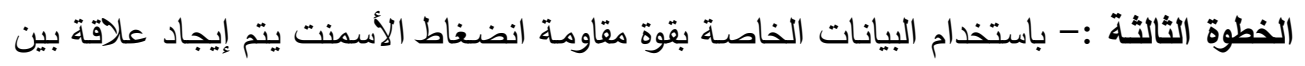

$$
\text { القوة وكل طور من الأطوار الأربعة. }
$$

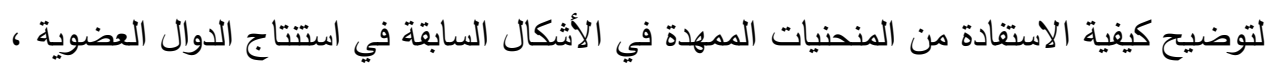

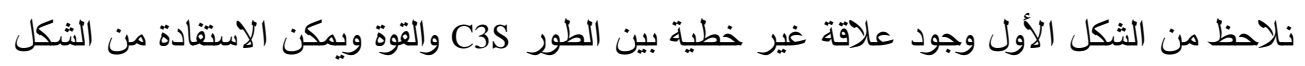

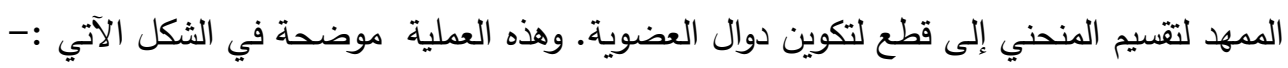

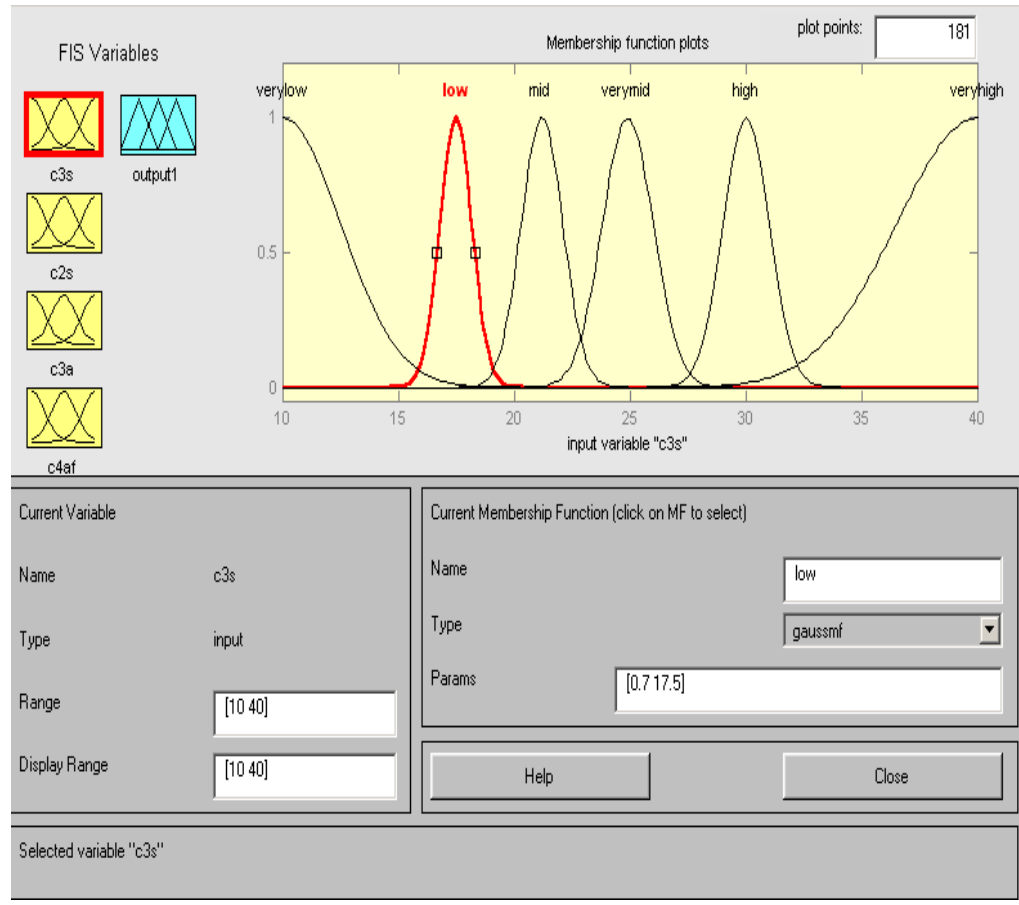

الثكل (5) الدالات العضوية للطور C3S 
في الثكل (5) تم رسم دوال العضوية اعتمادا على الثكل (1) ، إذ تم تقسيم المنحني إلى إنى ست قطع اعتمادا على قوة مقاومة انضغاط الإسمنت :القطعة الأولى سميت فيها الدالة العضوية (Very Low). القطعة الثانية سميت فيها الدالة العضوية (Low ). القطعة الثالثة سميت فيها الدالة العضوية (Mid) ). القطعة الرابعة سميت فيها الدالة العضوية (Very Mid ). القطعة الخامسة سميت فيها الدالة العضوية (High ). القطعة السادسة سميت فيها الدالة العضوية (Very High ). إن الدالة العضوية Very Low على سبيل المثال تكون اعظم قيمة لها، أي تكون درجة العضوية تساوي "1" ، عندما تكون قيمة C3S هي 10 وعندها تكون الدالة في ذروتها وكلما تزداد قيمة C3S تقل درجة العضوية وهذا هو الأساس الذي يستند إليه عمل دوال العضوية. ان المدى فئل للمتغير C3S بين 10 و40 وضمن هذا المدى تم رسم دوال العضوية.ان سبب استخدام دوال العضوية من نوع Gaussmf هو اقتراب المعدل الحسابي من الوسيط للأطوار الأربعة باستثناء طور C4AF لنتنقل الآن إلى العلاقة بين C2S وقوة مقاومـة الانضـاط. نلاحظ من الثكل (2) وجود علاقة غير خطية بين الطور C2S والقوة ويمكن الاستفادة من الثكل الممهد لتقسيم المنحني إلى الى قطع لتكوين دوال العضوية ـ وهذه القطع موضحة في الثكل الآتي :- 


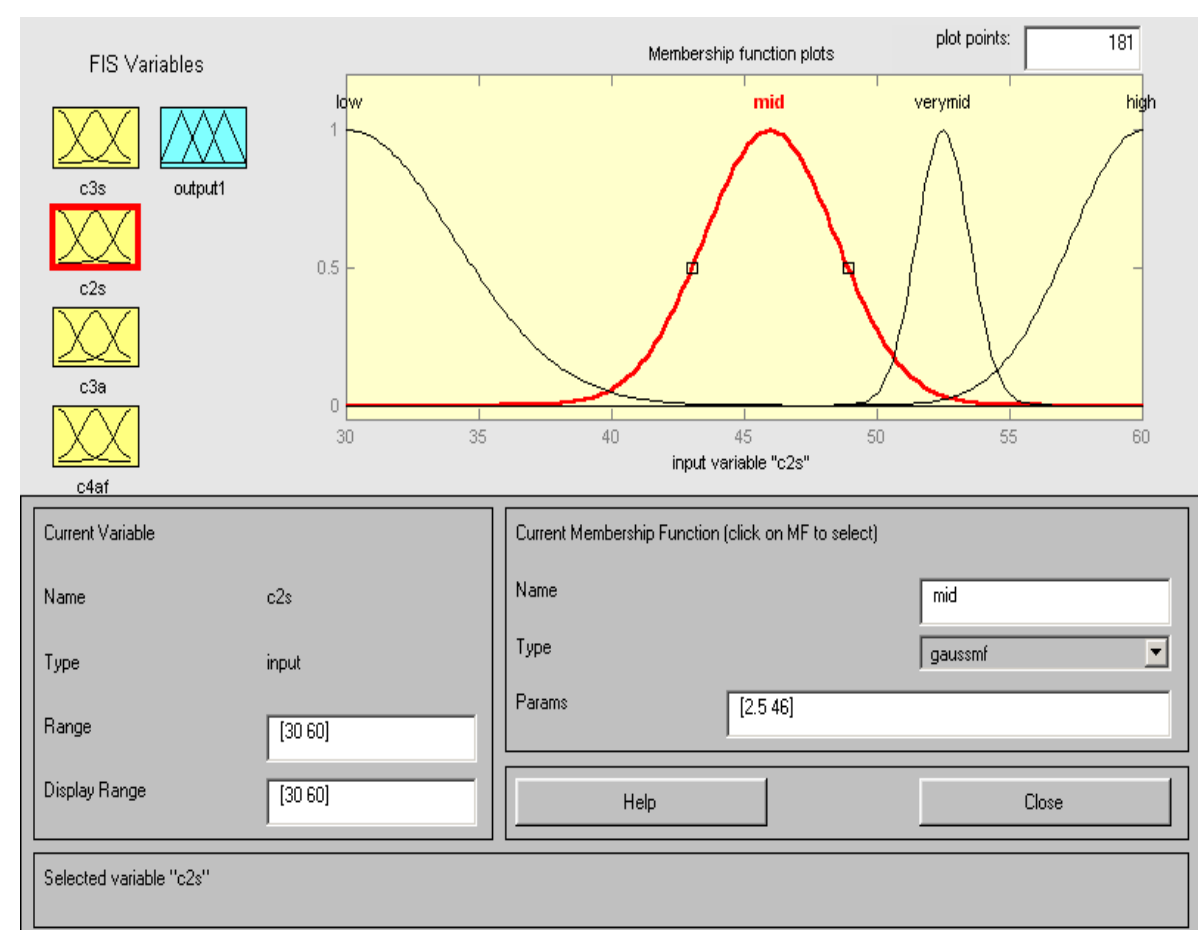

شكل (6) الدالات العضوية للطور

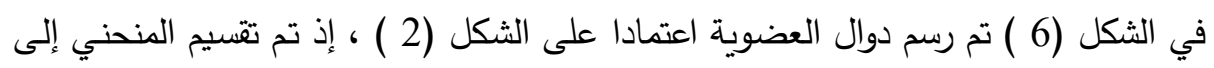
أربع قطع اعتمادا على قوة مقاومة انضغاط الإسمنت :القطعة الأولى سميت فيها الدالة العضوية (Low ) .

القطعة الثانية سميت فيها الدالة العضوية (Mid ). القطعة الثالثة سميت فيها الدالة العضوية (Very Mid ) . القطعة الرابعة سميت فيها الدالة العضوية (High ). إن الدالة العضوية Low على سبيل المثال تكون اعظم قيمة لها أي تكون درجة العضوية تساوي "1" ، عندما تكون قيمة C2S هي 60 وعندها تكون الدالة في ذروتها وكلما تقل قيمة

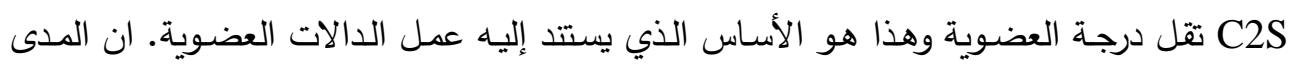
للمتغير C2S بين 30 و60 وضمن هذا المدى تم رسم دوال العضوية الأربعة. 
لو انتقلنا الآن إلى دراسة العلاقة بين C3A وقوة الانضغاط فأننا نلاحظ من الثكل (3 ) انه توجد

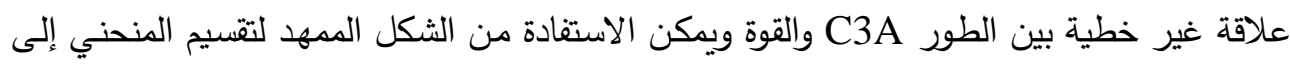

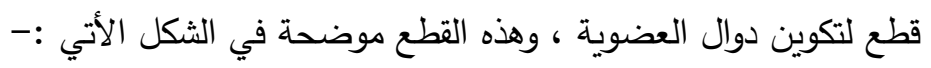

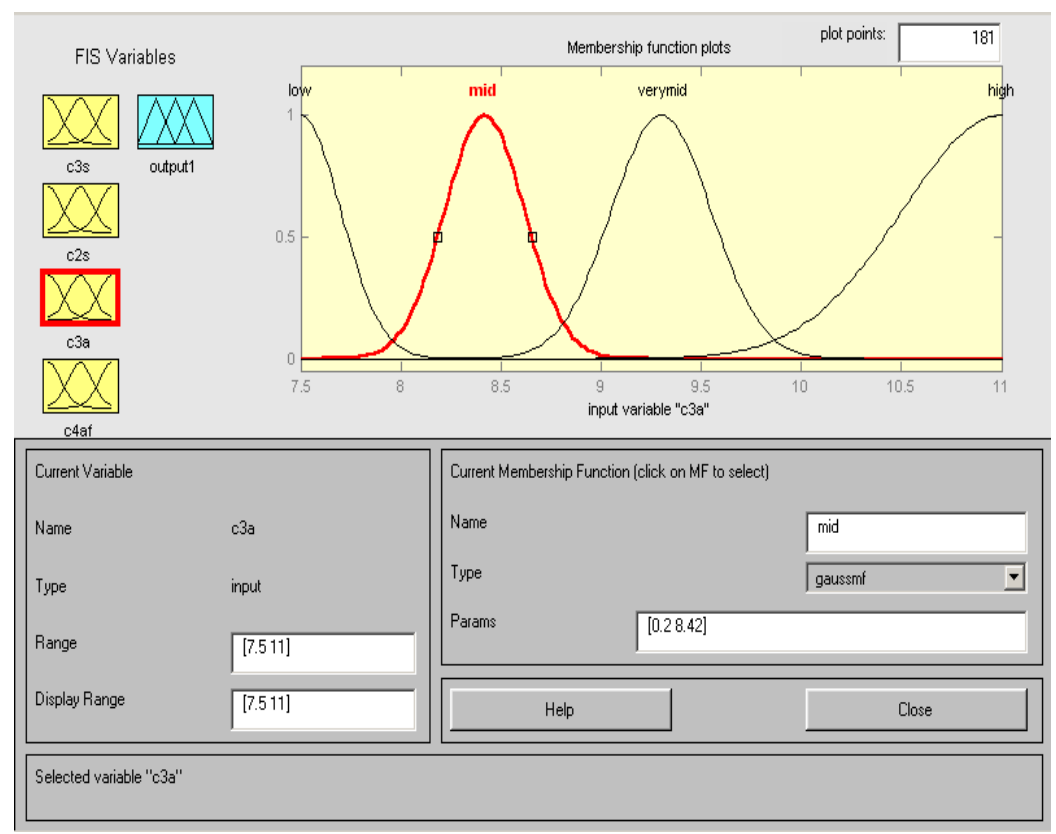

الثكل (7 ) الدالات العضوية للمتغير C3A

في الثكل (7 ) تم رسم دوال العضوية اعتمادا على الثكل (3) إذ تم تقسيم المنحني إلى إلى

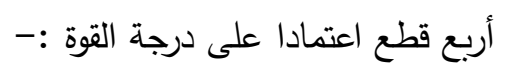

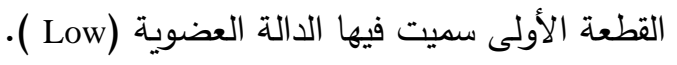

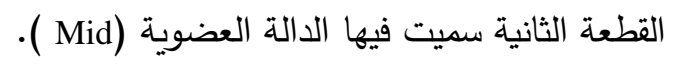
القطعة الثالثة سميت فيها الدالة العضوية (Very Mid ) ). القطعة الرابعة سميت فيها الدالة العضوية (High). ولدراسة العلاقة بين C4AF وقوة الانضغاط فإننا نلاحظ من الشكل (4 ) وجود علاقة غير خطية بين الطور C4AF والقوة ويمكن الاستفادة من الثكل الممهد لتقسيم المنحني الى قطع لتكوين

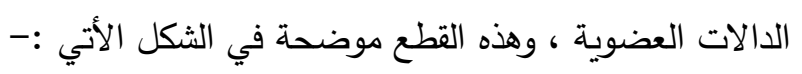




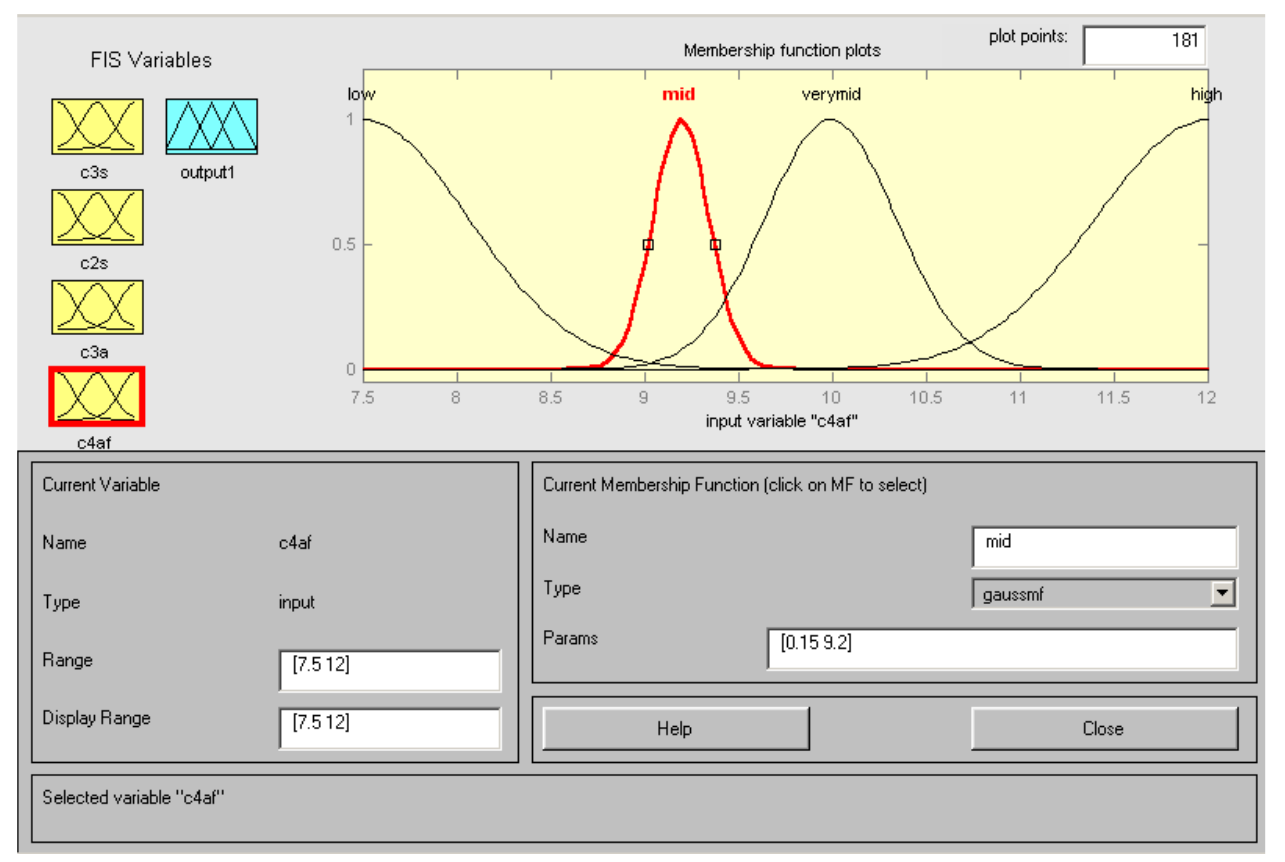

الثكل (8 ) الدالات العضوية للمتغير C4AF

في الثكل (8 ) تم رسم دوال العضوية اعتمادا على الثكل (4 ) إذ تم تقسيم المنحني إلى إلى

أربع قطع اعتمادا على درجة القوة :-

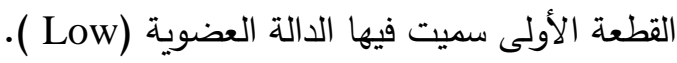

القطعة الثانية سميت فيها الدالة العضوية (Mid) ).

القطعة الثالثة سميت فيها الدالة العضوية (Very Mid ) .

القطعة الرابعة سميت فيها الدالة العضوية (High).

أخيرا يتم تقسيم الخارج output إلى عدد من دوال العضوية وحسب ما يقتضيه النظام

وكما موضح بالثكل الأتي :- 


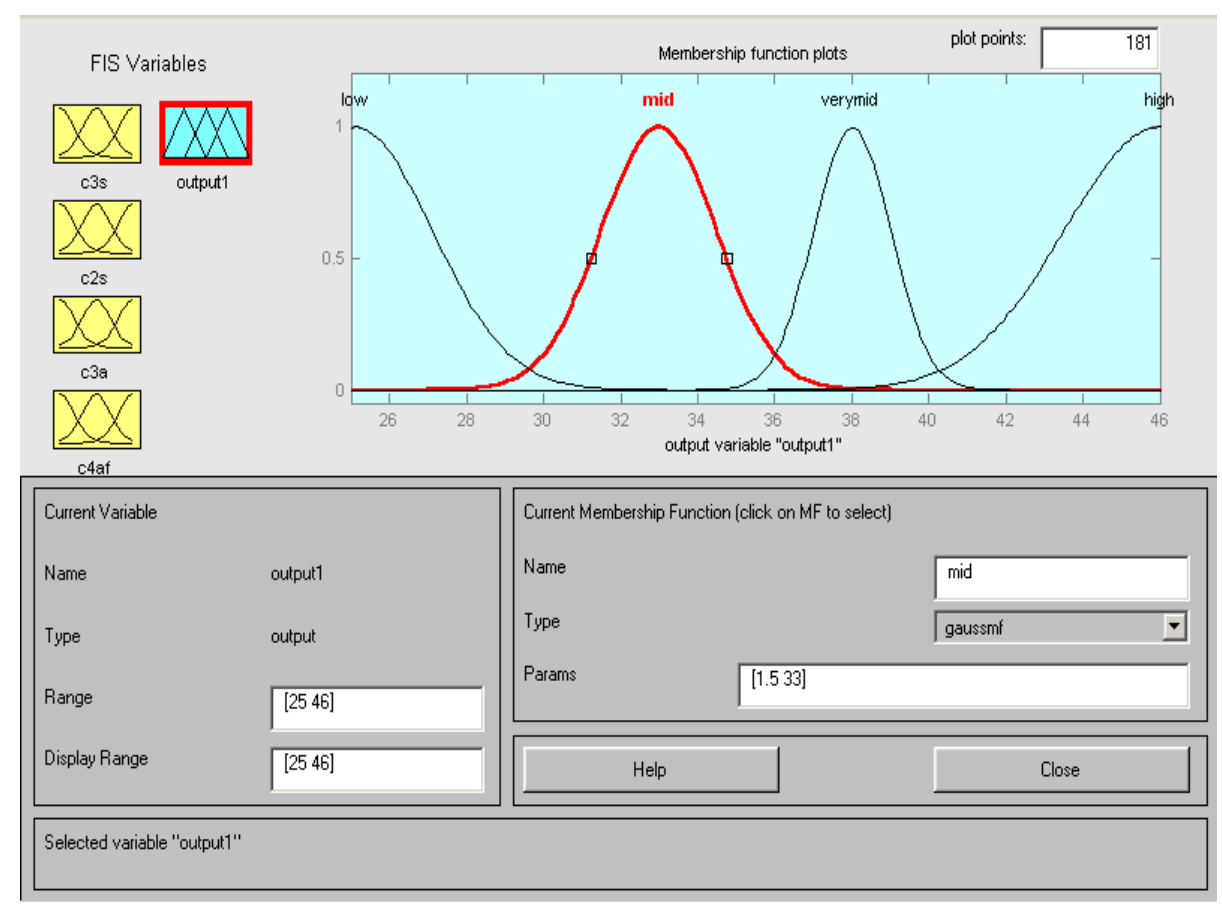

$$
\begin{aligned}
& \text { الشكل (9 ) الدالات العضوية لمتغير الإخراج } \\
& \text { في الثكل (9 ) تم تقسيم المنحني إلى أربع قطع اعتمادا على درجة قوة الانضغاط } \\
& \text { للإسمنت:- } \\
& \text { القطعة الأولى سميت فيها الدالة العضوية (Low ). } \\
& \text { القطعة الثانية سميت فيها الدالة العضوية (Mid) ). } \\
& \text { القطعة الثالثة سميت فيها الدالة العضوية (Very Mid). } \\
& \text { القطعة الرابعة سميت فيها الدالة العضوية (High). }
\end{aligned}
$$

الآن وبعد تصميم دوال العضوية لمتغيرات الإدخال والإخراج ورسمها نبدأ بتكوين القواعد العدات

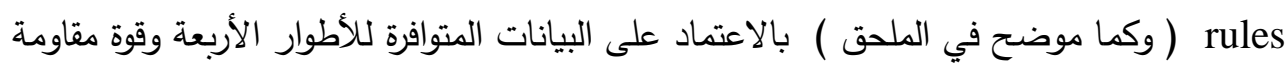

انضغاط الأسمنت فعلى سبيل المثال إذا كان كل من الأطوار الأربعة على النحو النيات الأتي :-

$\mathrm{C} 3 \mathrm{~S}=23.4$

$\mathrm{C} 2 \mathrm{~S}=47.2$

$\mathrm{C} 3 \mathrm{~A}=8.9$

$\mathrm{C} 4 \mathrm{AF}=11.1$ 


$$
\begin{aligned}
& \text { فإننا نحاول الآن ان نجد دالة العضوية التي تتتمي إليها كل قيمة من القيم السابقة.ويمكن }
\end{aligned}
$$

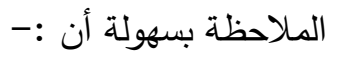

$$
\begin{aligned}
& \text { Cبنتمي إلى دالة العضوية التي اسمها Mid بدرجة معينة و Very Mid بدرجة معينة. } \\
& \text { تتنمي إلى دالة العضوية التي اسمها Mid C2S }
\end{aligned}
$$

إذ أن قيم الأطوار الأربعة وقيمة قوة مقاومة الأسمنت قد تم حسابها من قبل (المعاضيدي

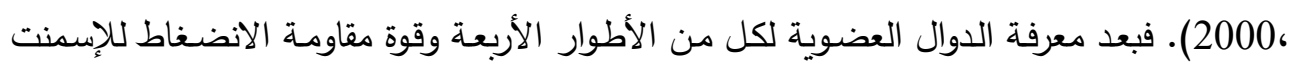

$$
\text { اصبح بالإمكان استتاج القواعد وعلى النحو الأتي :- }
$$

1. If [c3s is Very Mid] and [c2s is Mid] and [c3a is Hiah] and [c4af is Low] then [output1 is mid] [1]

2. If ( $\mathrm{c} 3 \mathrm{~s}$ is Mid) and (c2s is Mid) and (c3a is High) and (c4af is Low] then (output1 is mid) (1)

3. If (c3s is High) and (c2s is Mid) and (c3a is High) and (c4af is Low) then (output1 is Very_Mid) (1)

4. If [c3s is Very_Low] and (c2s is High) and (c3a is High) and (c4af is Low) then (output is low) (1)

5. If ( $\mathrm{c} 3 \mathrm{~s}$ is Very_Mid) and (c2s is Mid) and (c3a is High) and (c4af is Low) then (output1 is Very_Mid) (1)

6. If [ $\mathrm{c} 3 \mathrm{~s}$ is Very_High) and ( $\mathrm{c} 2 \mathrm{~s}$ is Low] and ( $\mathrm{c} 3 \mathrm{a}$ is Low] and (c4af is High) then (output1 is mid) (1)

7. If (c3s is Mid) and (c2s is Mid) and ( $\mathrm{c} 3 \mathrm{a}$ is High) and ( $\mathrm{c} 4 \mathrm{af}$ is Low) then (output is High) (1)

8. If (c3s is High) and (c2s is Low) and (c3a is High) and (c4af is Low) then (output1 is low) (1)

9. If (c3s is High) and (c2s is Low) and (c3a is High) and (c4af is Low) then (output is mid) (1)

10. If (c3s is Mid) and (c2s is Mid) and (c3a is High) and (c4af is Low] then (output1 is low) (1)

11. If (c3s is Mid) and (c2s is Very_Mid) and (c3a is High) and (c4af is Low] then (output is low] (1)

12. If [ $\mathrm{c} 3 \mathrm{~s}$ is Low] and [c2s is Very_Mid] and [c3a is High] and (c4af is Low) then [output1 is low] (1)

13. If ( $\mathrm{c} 3 \mathrm{~s}$ is High) and ( $\mathrm{c} 2 \mathrm{~s}$ is Mid) and [c3a is High) and ( $\mathrm{c} 4 \mathrm{af}$ is Low) then (output1 is mid) (1)

14. If [c3s is Mid] and [c2s is Mid] and [c3a is Hiahl and [c4af is Low] then loutput1 is Very Midl [1]

15. If ( $\mathrm{c} 3 \mathrm{~s}$ is Mid) and (c2s is Mid) and (c3a is Mid) and (c4af is High) then (output1 is mid) (1)

16. If (c3s is Mid) and (c2s is Mid) and (c3a is Very_Mid) and (c4af is High) then (output1 is mid) (1)

17. If (c3s is Very_Mid) and (c2s is Mid) and (c3a is Mid) and (c4af is High) then (output1 is mid) (1)

18. If (c3s is Very_Mid) and (c2s is Mid] and (c3a is Very_Mid) and (c4af is High) then (output1 is mid) (1)

19. If (c3s is Very_Mid) and (c2s is Mid) and (c3a is Mid) and (c4af is Very_Mid) then (output1 is Very_Mid) (1)

20. If (c3s is Very_Mid) and (c2s is Mid) and (c3a is Mid) and (c4af is High) then (output is Very_Mid) (1)

21. If [ $\mathrm{c} 3 \mathrm{~s}$ is High] and (c2s is Mid) and (c3a is Mid) and (c4af is Very_Mid) then (output1 is Very Mid) (1)

22. If ( $\mathrm{c} 3 \mathrm{~s}$ is High) and (c2s is Mid) and (c3a is Mid) and ( $\mathrm{c} 4 \mathrm{af}$ is High) then (output1 is Very_Mid] (1)

23. If (c3s is Very_Mid) and (c2s is Mid) and (c3a is Mid) and (c4af is Very_Mid) then (output1 is High) (1)

24. If ( $\mathrm{c} 3 \mathrm{~s}$ is Very_Mid) and ( $\mathrm{c} 2 \mathrm{~s}$ is Mid) and ( $\mathrm{c} 3 \mathrm{a}$ is Mid) and (c4af is High) then (output1 is High) (1)

25. If (c3s is High) and (c2s is Mid) and (c3a is Mid) and (c4af is Very_Mid) then (output1 is High) (1)

26. If ( $\mathrm{c} 3 \mathrm{~s}$ is High) and (c2s is Mid) and (c3a is Mid) and (c4af is High) then (output is High) (1)

27. If (c3s is Very_Mid) and (c2s is Low] and (c3a is Mid) and (c4af is Very_Mid) then (output 1 is Very_Mid) (1)

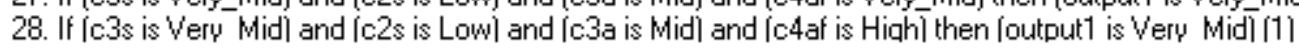


29. If (c3s is High) and (c2s is Low] and (c3a is Mid) and (c4af is Very_Mid) then (output1 is Very_Mid) (1) 30. If ( $\mathrm{c} 3 \mathrm{~s}$ is High) and ( $\mathrm{c} 2 \mathrm{~s}$ is Low] and ( $\mathrm{c} 3 \mathrm{a}$ is Mid) and (c4af is High) then (output 1 is Very_Mid) (1) 31. If (c3s is Very_Mid) and (c2s is Low] and (c3a is Mid) and (c4af is Very_Mid) then (output1 is High) (1) 32. If (c3s is Very_Mid) and (c2s is Low) and (c3a is Mid) and (c4af is High) then (output 1 is High) (1) 33. If [ $\mathrm{c} 3 \mathrm{~s}$ is High) and ( $\mathrm{c} 2 \mathrm{~s}$ is Low] and (c3a is Mid) and (c4af is High) then (output1 is High) (1) 34. If ( $\mathrm{c} 3 \mathrm{~s}$ is High) and (c2s is Low) and (c3a is Mid) and (c4af is Very_Mid) then (output is High) (1) 35. If ( $\mathrm{c} 3 \mathrm{~s}$ is Mid) and (c2s is Mid) and (c3a is Mid) and (c4af is Very_Mid) then (output1 is High) (1) 36. If ( $\mathrm{c} 3 \mathrm{~s}$ is Low) and (c2s is Mid) and (c3a is Mid) and (c4af is Very_Mid) then (output1 is High) (1) 37. If (c3s is Very_Mid) and (c2s is Low] and (c3a is High) and (c4af is Low) then (output1 is low] (1) 38. If (c3s is Very_Mid) and (c2s is Mid) and (c3a is High) and (c4af is Low) then (output1 is low) (1) 39. If ( $\mathrm{c} 3 \mathrm{~s}$ is High) and (c2s is Mid) and (c3a is High) and (c4af is Low) then (output1 is low] (1) 40. If (c3s is Very_Low) and (c2s is High) and (c3a is High) and (c4af is Low) then (output1 is mid) (1) 41. If ( $\mathrm{c} 3 \mathrm{~s}$ is Mid) and ( $\mathrm{c} 2 \mathrm{~s}$ is Mid) and ( $\mathrm{c} 3 \mathrm{a}$ is Very_Mid) and ( $\mathrm{c} 4 \mathrm{a}$ is Low) then (output1 is low) (1) 42. If ( $\mathrm{c} 3 \mathrm{~s}$ is Low] and (c2s is Mid) and (c3a is High) and ( $\mathrm{c} 4$ af is Low) then (output 1 is low) (1)

43. If (c3s is Low] and (c2s is Mid) and (c3a is High) and ( $c 4$ af is Low] then [output1 is mid) (1) 44. If (c3s is Low) and (c2s is Very_Mid) and (c3a is High) and (c4af is Low) then (output1 is mid) (1) 45. If ( $\mathrm{c} 3 \mathrm{~s}$ is Mid) and (c2s is Very_Mid) and (c3a is High) and (c4af is Low] then (output1 is mid) (1) 46. If (c3s is Very_Low) and (c2s is High) and (c3a is Very_Mid) and (c4af is Low) then (output1 is mid) (1) 47. If (c3s is Very_Low) and (c2s is High) and (c3a is Very_Mid) and (c4af is Low) then (output1 is low) (1) 48. If [ $\mathrm{c} 3 \mathrm{~s}$ is Low] and ( $\mathrm{c} 2 \mathrm{~s}$ is High) and (c3a is High) and ( $\mathrm{c} 4 \mathrm{a}$ ) is Low] then (output1 is mid] (1) 49. If [ $\mathrm{c} 3 \mathrm{~s}$ is Low] and (c2s is High) and [ $\mathrm{c} 3 \mathrm{a}$ is High) and [c4af is Low] then (output1 is low] (1) 50. If [ $c 3$ s is Very_Low] and [c2s is High] and (c3a is High) and (c4af is Mid] then (output1 is low] (1) 51. If (c3s is Very_Low) and ( $\mathrm{c} 2 \mathrm{~s}$ is High) and (c3a is High) and (c4af is Mid) then (output1 is mid) (1) 52. If ( $\mathrm{c} 3 \mathrm{~s}$ is Low] and [c2s is High) and [c3a is High) and (c4af is Mid] then (output1 is low] (1) 53. If ( $\mathrm{c} 3 \mathrm{~s}$ is Low) and (c2s is High) and (c3a is High) and (c4af is Mid) then (output is mid) (1) 54. If (c3s is Very_Mid) and (c2s is Mid) and (c3a is Low) and (c4af is High) then (output1 is mid) (1) 55. If ( $\mathrm{c} 3 \mathrm{~s}$ is Very_Mid) and (c2s is Mid) and (c3a is Low) and (c4af is High) then (output 1 is Very_Mid) (1) 56. If [c3s is Very Highl and [c2s is Lowl and [ $\mathrm{c} 3 \mathrm{a}$ is Midl and [ $\mathrm{c} 4 \mathrm{af}$ is Hiahl then loutput1 is mid [1] 57. If (c3s is Very_High) and (c2s is Low] and (c3a is Mid) and (c4af is High) then (output1 is low] (1) 58. If (c3s is Very_High) and (c2s is Low) and (c3a is Low) and (c4af is High) then (output1 is low] (1) 59. If (c3s is Very_Mid) and (c2s is Mid) and (c3a is Mid) and (c4af is Very_Mid) then (output1 is mid) (1) 60. If (c3s is Very_Mid) and (c2s is Mid] and (c3a is Low] and (c4af is Very_Mid) then (output1 is mid] (1) 61. If (c3s is Very_Mid) and (c2s is Mid) and (c3a is High) and (c4af is Mid) then (output1 is mid) (1) 62. If [ $\mathrm{c} 3 \mathrm{~s}$ is Very_Mid) and (c2s is Mid) and [c3a is High) and (c4af is Mid) then (output1 is low] (1) 63. If (c3s is Very_Mid) and (c2s is Mid) and (c3a is High) and (c4af is Mid) then (output1 is Very_Mid) (1) 64. If [c3s is Very Mid) and [c2s is Mid) and [c3a is Highl and [c4af is Low] then loutput1 is Hiahi [1]

$$
\begin{aligned}
& \text { بعد تكوين القواعد وإظهار النتائج نعمل على إزالة الضبابية ونحصل على النتيجة النهائية } \\
& \text { أي نحصل على قوة مقاومة انضغاط الإسمنت وهذه القيمة تتم مقارنتها مع القيمة المحسوبة لإظهار } \\
& \text { الأسلوب الأفضل وكما موضتح في الجدول (4). } \\
& \text { 5. تطبيق النظام والنتائج :- } \\
& \text { يتم إدخال البيانات التي تمثل التحاليل الكيمائية للإِسمنت إلى النظام وذلك عن طريق } \\
& \text { الواجهة المصممة لهذا الغرض ، إذ يتم حساب الأطوار الأربعة ، وبعدها يتم الدخول إلى النظام } \\
& \text { المضبب الذي يكون في ملف اسمه D_C_S.fis و إعطائه إلى متغير معين وليكن Sys ، وبعد } \\
& \text { ذلك يتم استخدام الدالة ( evalfis ) وهذه الدالة تحتوي على متغيرين الأول يمثل المدخلات إلى } \\
& \text { النظام والثاني يمثل المتغير الخاص بالنظام وكما يأتي :- } \\
& \text { sys =readfis( ("D_C_S.fis “) }
\end{aligned}
$$


comp_str=evalfis ([C3S C2S C3A C4AF ] , sys );

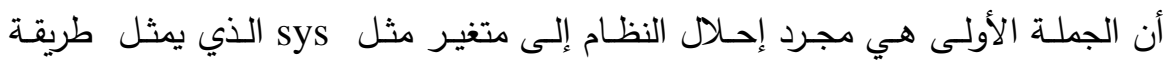

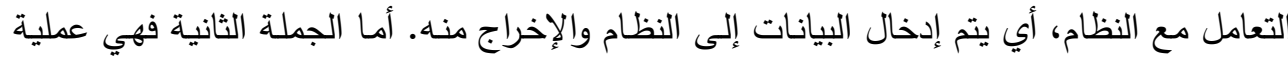

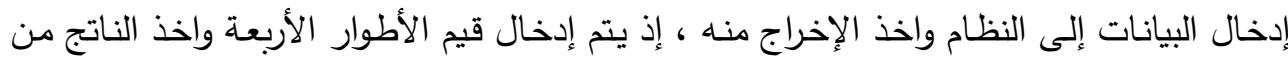
المتغير Comp_Str ـ فعن طريق دالات العضوية والقواعد المكونة يتم اخراج النتائج.ولو أدخلنا

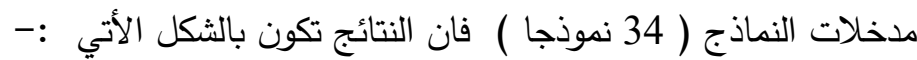

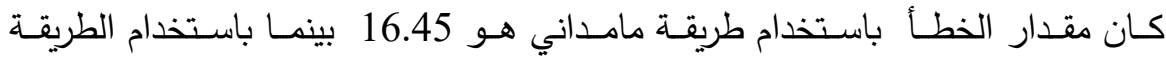

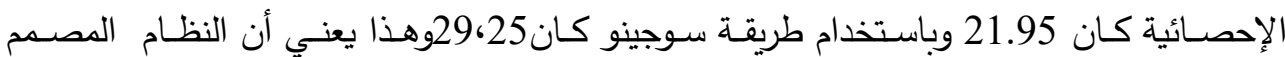

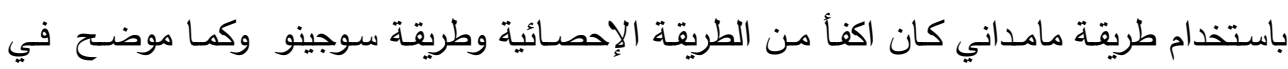

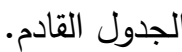

الجدول (1) يوضح تباين درجة قوة مقاومة انضغاط الإسمنت بالطرائق الثلاثة

\begin{tabular}{|c|c|c|c|c|}
\hline طريقة سوجينو & طريقة مامداني & الطريقة الإحصائية & القيم الحقيقية & تسلسل النموذج \\
\hline 32.2 & 33 & 31.4 & 37.5 & 1 \\
\hline 35.4 & 35.5 & 31.4 & 38 & 2 \\
\hline 35.4 & 35.5 & 31.4 & 35.5 & 3 \\
\hline 34.8 & 34.5 & 34.9 & 32 & 4 \\
\hline 41.9 & 40.2 & 35.2 & 38.5 & 5 \\
\hline 45.6 & 42.4 & 35.1 & 43 & 6 \\
\hline 30.7 & 32.7 & 32.3 & 25 & 7 \\
\hline 35.4 & 35.5 & 32.3 & 34.5 & 8 \\
\hline 29.6 & 31.4 & 32.3 & 33.5 & 9 \\
\hline 29 & 30.6 & 32.5 & 28.5 & 10 \\
\hline 35 & 35.5 & 33.1 & 27.5 & 11 \\
\hline 32 & 34.1 & 31.6 & 28.5 & 12 \\
\hline 29 & 30.7 & 31.5 & 30 & 13 \\
\hline 29 & 30.9 & 32.4 & 28.5 & 14 \\
\hline 35.4 & 31 & 32.3 & 29.5 & 15 \\
\hline 29 & 35 & 35.2 & 35.5 & 16 \\
\hline 36.5 & 30.6 & 34.5 & 31.5 & 17 \\
\hline 33 & 36 & 34.5 & 34.5 & 18 \\
\hline 34.6 & 33.8 & 32.7 & 30.5 & 19 \\
\hline 35.4 & 35.5 & 32.8 & 37 & 20 \\
\hline 29.1 & 35.5 & 32.8 & 45.5 & 21 \\
\hline 29.2 & 31.7 & 32.7 & 28.5 & 22 \\
\hline 32.9 & 33.2 & 33.3 & 30.5 & 23 \\
\hline 31 & 34 & 32.7 & 28.5 & 24 \\
\hline 30 & 33.1 & 32.7 & 33.5 & 25 \\
\hline
\end{tabular}




\begin{tabular}{|c|c|c|c|c|}
\hline 30.2 & 33.1 & 32.7 & 30.5 & 26 \\
\hline 33.4 & 34.9 & 33 & 34.5 & 27 \\
\hline 29.6 & 31.6 & 35.7 & 36.5 & 28 \\
\hline 29 & 30.7 & 36.6 & 40 & 29 \\
\hline 32.9 & 33 & 34 & 33 & 30 \\
\hline 37.9 & 37.7 & 34.7 & 41 & 31 \\
\hline 38.9 & 37.9 & 35.4 & 31.5 & 32 \\
\hline 39.1 & 37.7 & 34.8 & 34 & 33 \\
\hline 34.2 & 34.3 & 34.3 & 32.5 & 34 \\
\hline
\end{tabular}

إن العلاقـة بين قوة انضـغاط الأسمنت وكل مـن الأطـوار الأربعـ C3S و C2S و

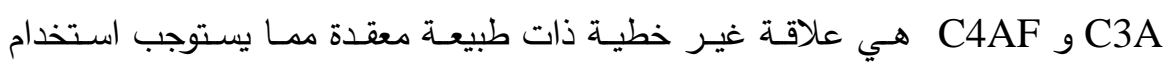
أساليب رياضية كفؤهة للتعامل مع مثل هذه العلاقات. إن اختيار أسلوب المنطق المضبب يبدو موضوعيا لان هذا الأسلوب مخصص هص أصلاه لمعالجة النظم المعقدة. - مان.

إن النظام الحاسوبي الذي تم إعداده يمكن الاستفادة منه من قبل معامل الإسمنت من

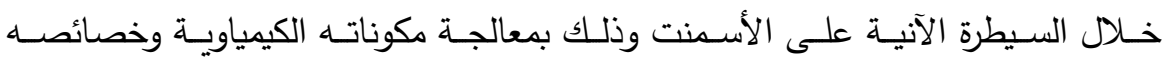
الفيزياويـة مـع المواصفات القياسية. كذلك فان النظام يقدم للعاملين في الإسمنت تقديرا مقبولا لقوة مقاومة انضغاط الإسمنت بعد (28) يوما. 7.

يمكن تطوير العمل الحالي باتجاهات مختلفة ـ ولعل من التوصيات الملحة هو تطوير النظام لتقدير قوة الانضغاط بعد أية مدة زمنية ( غير محددة) وبطبيعة الحال فان مثل هذا

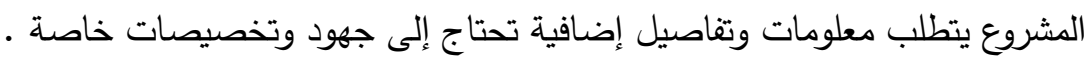
ومن التوصيات العامة فإننا نوصي بإنشاء مركز تخصصي لدراسة الإسمنت العراقي في .2 جوانبه كافة بما في ذلك استخدامات المنطق المضبب في عمليات التصنيع والسيطرة النوعية.

\section{المصادر}

حمودات ، عابدة تحسين توفيق (2002) ، "استخدام المنطق المضبب في صنع القرار لتخمين تسعير منتوج جديد " ، رسالة ماجستير ، جامعة الموصل ، ل 


$$
\begin{aligned}
& \text { الدباغ ، سالم محمود والمعاضيدي ، ساهرة محمد عثمان (2004) ، "العوامل المؤثرة في } \\
& \text { الخواص الفيزيائية للإسمنت البورتلندي العراقي " ، مجلة علوم الرافدين العدد (1) المجلد }
\end{aligned}
$$

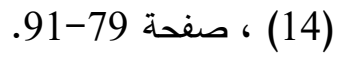

$$
\begin{aligned}
& \text { ذنون ، باسل يونس (1998) ، "التقدير اللبي : أسلوب بياني في التقدير الإحصائي " ، }
\end{aligned}
$$

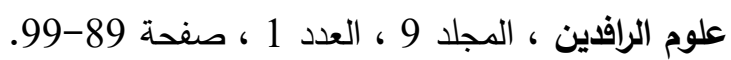

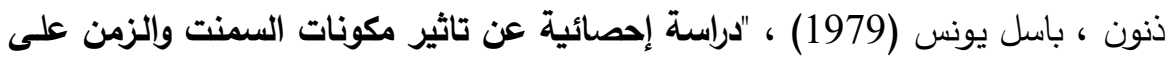

$$
\begin{aligned}
& \text { صلابته " ، جامعة الموصل ، صفحة 3-20 لئل } \\
& \text { المعاضيدي ، سـاهرة محمد عثـان و الدباغ ، سـالم محمود (2001) ، مـلولولات تقدارير }
\end{aligned}
$$

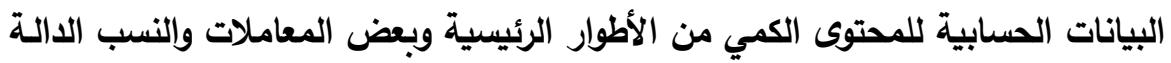

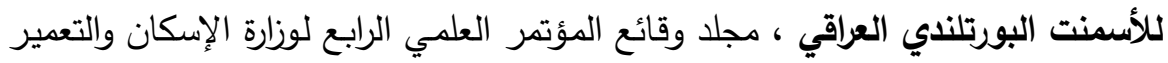

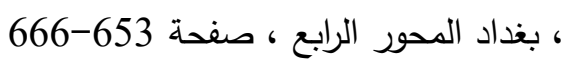

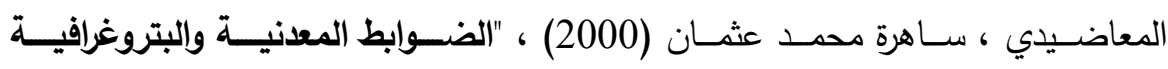

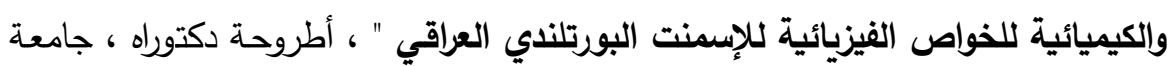

$$
\begin{aligned}
& \text { الموصل ، صفحة 103-167 }
\end{aligned}
$$

[7] Bogue (1929), In : Lea, F . M. (1965) The chemistry of cement and concrete , Edward Arnold (publishers) ltd, London , $2^{\text {th }}$ edition , pp.184-211

[8] Kandel, Abrahams, (1986), Fuzzy Mathematical Techniques with Applications , Addison - Wesley publishing company .

[9] Kohlhaas, B.et al(1983), Cement Engineers Handbook, Fourth English edition, originated by Otto Labahn, translated by C. van Amerongen from the sixth German edition , Bauverlag Gmbh, Wiesbaden and Berlin, pp.625-653.

[10] Ruan , da, (1997), Intelligent Hybrid Systems : Fuzzy Logic , Neural Networks and Genetic Algorithms, Klwer Academic publishers, pp.21-32.

[11] Zadeh Lotfi a., (1995), Fuzzy Logic Toolbox, Berkerly, pp.3-82. 